\title{
Secure Data Retrieval for Decentralized Military Networks
}

\author{
Karishma S. Dule \\ Research Student \\ Department of Computer Science \\ Government College of Engineering. \\ Aurangabad, Maharashtra
}

\author{
M.B. Nagori \\ Assistant Professor \\ Department of Computer Science \\ Government College of Engineering. \\ Aurangabad, Maharashtra
}

\begin{abstract}
Portable nodes in military environments, for example, as in battlefield or an unfriendly area are prone to experience the under go of irregular system network and frequent partitions. Interruption tolerant network (ITN) innovations are getting to be fruitful results that permit remote device conveyed by officers to speak with one another and access the secret data or summon dependably by abusing outside capacity nodes. Possibly the most solid issues in this situation are the necessity of approval arrangements and the strategies redesign for secure information recovery.

This paper considers an attribute-based secure data retrieval scheme using $\mathrm{CP}-\mathrm{ABE}$ for ITNs where multiple key authorities manage their attributes independently. Immediate attribute repeal enhances backward/forward secrecy of confidential data by reducing the windows of vulnerability. Key escrow problem is resolved by an escrow-free key issuing protocol that utilizes the characteristic of the decentralized ITN architecture proposed a decentralized approach; their technique does not authenticate users. We demonstrate how to apply the proposed mechanism to securely and efficiently manage the confidential data distributed in the Interruption-tolerant military network.
\end{abstract}

\section{Keywords}

Access-control, Attribute-based encryption (ABE), Interruption-tolerant Network (ITN), Multiauthority, Secure Data Retrieval.

\section{INTRODUCTION}

In military system environment, associations of remote gadgets conveyed by officers may be briefly detached by sticking, ecological variables, and versatility, particularly when they work in hostile environments. Interruption tolerant system (DTN) technologies are becoming favorably result that authorize nodes to communicate with each other in these immensely networking environments. [1]-[3].Naturally, when there is no limit to-end attachment between a source and a destination pair, the messages from the source node may need to wait in the middle nodes for a considerable amount of time until the connection would be finally established. Roy [4] and Chuah [5] presented capacity hubs in ITNs where information is put away or duplicated such that just approved movable hubs can get to the essential data rapidly and completely. Innumerable military applications require enlarge security of private information including access control procedure that are cryptographically implemented. In many cases, it is sensible to provide discriminate access services such that data access policies are defined over user attributes or roles, which are control by the key authorities. For illustration, in a
Interruption-tolerant military network, a commander may store classified data at a stockpiling hub, which ought to be gotten to by parts of "Legion 1" who are partaking in "District 2." It is a reasonable assumption that multiple key authorities are likely to manage their own dynamic attributes for soldiers in their deployed regions, which could be frequently changed (e.g., the attribute representing current location of moving soldiers) [4], [8], [9].We suggest to this ITN construction where various powers issue and deal with their trait keys freely as a decentralized ITN [10]. The idea of attribute based encryption (ABE) [11]-[14] is an encouraging approach that satisfies the necessities for secure information recovery in ITN.

$\mathrm{ABE}$ characteristics an instrument that authorize a right to gain approach control over scrambled information utilizing access approaches and attributed qualities among private keys and cipher text. Mostly, cipher text-policy ABE (CP-ABE) provides a expandable way of encrypting data such that the encryptors defines the attribute set that the decrypted needs to possess in order to decrypt the cipher text [13]. Thus, dissimilar users are allowed to decrypt dissimilar pieces of data according to the security policy.

On the other hand, the ABE to ITN presents a few security and protection challenges. Since a small number of clients may change their related qualities eventually (for instance, moving their area), or some private keys might be compromised, key repeal (or update) for each attribute is necessary in order to make systems secure. This involves that repeal of any attribute or any single user in an attribute group would affect the other users in the group. For illustration, if a user add or quit an attribute group, the related attribute key should be changed and reconstruct to all the other members in the same group for backward or forward secrecy. It may result in congestion during rekeying procedure or security humiliation due to the windows of powerlessness if the previous attribute key is not updated immediately. An additional problem is the key escrow problem. In CP-ABE, the key authority produce private keys of users by exercise the authority's master secret keys to users' associated set of attributes. In this manner, the key control can decode each cipher text tended to particular clients by producing their attribute keys. If the key authority is damage by opponent when deployed in the hostile environments, this could be a potential threat to the data confidentiality or privacy especially when the data is highly sensitive. The key escrow is an built-in problem even in the various-authority systems as long as each key authority has the whole right to generate their own attribute keys with their own master secrets. The final problem is the coordination of attributes issued from dissimilar authorities. 


\section{RELATED WORKS}

$\mathrm{ABE}$ comes in two savor called key-policy ABE (KP-.ABE) and Cipher text policy attribute-based encryption. In KP-ABE the encryptors just gets to name a cipher text with a set of attributes. The key power picks an approach for each one client that figures out which cipher text he can unscramble and issues the way to every client by inserting the strategy into the client's key. The key authority chooses a policy for each user that decides which cipher text he can decrypt and issues the key to each user by embedding the policy into the user's key.. In CP-ABE, the cipher text is encrypted with an access policy chosen by an encryptors, but a key is simply created with respect to an attributes set. $\mathrm{CP}-\mathrm{ABE}$ is more appropriate to DTNs than KP-ABE because it enables encryptors such as a commander to choose an access policy on attributes and to encrypt confidential data under the access structure via encrypting with the corresponding public keys or attributes [4], [7].

1) Attribute Repeal: Bettencourt et al. [13] and Boldyreva et al. [16] first suggested key repeal structure in CP-ABE and $\mathrm{KP}-\mathrm{ABE}$. Their results are to adjoin to each attribute an termination date (or time) and spread a new set of keys to valid users after the termination. The regularly property revocable ABE plans [8], [13], have two primary issues. The first problem is the security humiliation in terms of the backward and forward confidentiality. It is a trustworthy situation that clients, for example, fighters may change their qualities frequently, e.g., position or area move when considering these as characteristics [4], [9].Then, a user who just envelop the attribute might be able to access the foregoing data encrypted before he obtains the attribute until the data is re-encrypted with the newly updated attribute keys by periodic rekeying (backward confidentiality).

2) Key Escrow: Most of the live ABE schemes are build on the architecture where a single trusted authority has the power to create the whole private keys of users with its master secret statistics [11], [13]. Thus, the key escrow problem is built-in such that the key authority can decrypt every cipher text approach to users in the system by creating their secret keys at any time. Chase et al. introduce a distributed KP-ABE scheme that solves the key escrow problem in a Multiauthority system.

\section{EXISITING FRAMEWORK}

The scheme of Attribute based encryption (ABE) is a promise approach that fulfills the requirement for secure information recovery in ITNs. ABE characteristics a system that permit a right to gain entrance control over scrambled information utilizing access approaches and credited qualities among private keys and cipher text. The issue of applying the ABE to ITNs presents a few security and protection challenges. Since a few clients may change their related qualities sooner or later (for instance, moving their district), or some private keys may be traded off, key repudiation (or redesign) for each one characteristic is fundamental keeping in mind the end goal to make frameworks secure. This infers that renouncement of any property or any single client in a characteristic gathering would influence alternate clients in the gathering. Case in point, if a client joins or leaves a trait assemble, the related characteristic key ought to be changed and redistributed to the various parts in the same gathering for retrograde or forward mystery. It may bring about bottleneck amid rekeying method or security corruption because of the windows of powerlessness if the past characteristic key is not overhauled quickly.

\section{PROPOSED FRAMEWORK}

In this paper, we suggest an attribute-based secure data recovery scheme utilizing $\mathrm{CP}-\mathrm{ABE}$ for decentralized ITNs. The proposed plan features the following attainment. Firstly, immediate attribute repeal enhances backward/forward secrecy of confidential data by reducing the windows of helplessness. Second, encryptors can characterize a finegrained access strategy utilizing any similarity access structure under feature issued from any chosen set of power. Third, the key escrow problem is resolved by a without escrow key issuing protocol that utilize the feature of the decentralized DTN structural engineering. The key issuing deals create and issues user secret keys by performing a reliable two-party processing (2PC) protocol among the key power with their own master secrets. The $2 \mathrm{PC}$ protocol intercept the key power from obtaining any master confidential information of each other such that none of them could create the whole set of user keys alone. Eventually, user are not needed to entirely believe the dominant presences keeping in mind the end goal to confidential their information to be convey. The information confidentiality and security might be cryptographically imposed against any disquisitive key power or information storage hubs in the proposed scheme.

\subsection{Preferences of Proposed Framework}

- Data confidentiality: Uncertified users who do not have enough accreditations rewarding the right to gain entrance approach ought to be prevented from getting to the plain data in the storage hub. Likewise, uncertified access from the storage node or key power ought to be additionally avoided.

- Collusion-safety: If numerous users collaborate, they may be able to decrypt a cipher text by combining their attributes even if each of the users cannot decrypt the cipher text alone [11]-[13].For illustration, suppose there occur a user with attributes \{"Battalion 1", "Region 1"\} and another user with attributes \{"Battalion 2", "Region 2"\}. They may advance in decrypting a cipher text encrypted under the access policy of ("Battalion 1" AND "Region 2"), even if each of them cannot decrypt it individually. We do not want these colluders to be able to decrypt the secret data by integrate their attribute.

- Backward and Forward Confidentiality: In the surroundings of $\mathrm{ABE}$, backward confidentiality implies that any user who comes to hold an attribute (that fulfill the access policy) should be stop from accessing the plaintext of the foregoing information interchange before he holds the attribute. Additionally, forward secrecy implies that any user who drops an attribute should be stop from accessing the plaintext of the consequent information interchange after he drops the attribute, unless the other defensible attributes that he is holding fulfill the right to satisfy the policy.

\section{FUNCTIONING OF FRAMEWORK}

- Key Authorities: They are key creation centers that create open/secret parameters for CP-ABE. The key authorities comprise of a central authority and numerous neighborhood authorities. We suppose that there are reliable and dependable transmission channels between a central authority and each 
neighborhood authority during the starting key setup and creation phase. All neighborhood authority superintends various attributes and issues corresponding attribute keys to users. They allow dissimilar access rights to individual users based on the users attributes dissimilar access rights to individual users based on the users attributes. The key authorities are assumed to be sincere-but interesting

- Storage hub: This is an organization that stores data from senders and give corresponding access to users. It may be mobile or fixed [4], [5]. Alike to the previous schemes, we also suppose the storage hub to be semi- trusted that is sincere-but interesting.

- Sender: This is an organization who claims personal messages or information (e.g., a commander) and wishes to store them into the external data storage hub for ease of sharing or for reliable distribution to users in the ultimate networking situations. A sender is charge for determine (attribute based) access arrangement and impose it on its own data by encrypting the data under the policy before putting away it to the storage hub.

- User: This is a mobile hub who wants to access the data stored at the storage hub (e.g., a fighter).If a user hold a set attributes fulfilling the access policy of the encrypted information characterized by the sender, and is not cancel in any of the attributes, then he will have the capacity to decrypt the cipher text and get the information.

\subsection{CP-ABE Policy}

In Cipher text Approach Quality based Encryption plot, the encryptors can transform the settlement, which can decrypt the scrambled message. The scheme could be organize with the assistance of attribute. We suggest a system in which access policy approach require not be sent alongside the cipher text, by which we have the ability safeguard the safety of the encryptors. This methods encrypt data might be kept classified regardless of the fact that the storage server is entrusted; besides, our method are secure against intrigue attack. Attribute Based Encryption structure utilized credits to portray the encrypt data and integrate arrangements with users keys; while in our structure attribute are utilized to represent a users qualifications, and a collecting encrypt data decides an arrangement for who can unscramble.

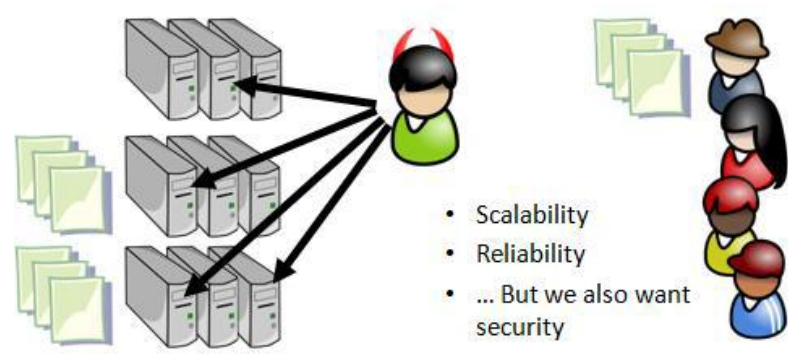

Fig1. Remote File Storage: Interesting Challenges

So one feature we have likelihood to do all time is store our files on remote servers. We have a tendency to might want to supply scalable access to our files to others victimization further resources on the market elsewhere-we have a likelihood to might want a lot of dependability just in case of failures. During this case we have likelihood to might want to equivalent our files totally dissimilar data centers or with dissimilar organizations. However we would like safety. We have a likelihood to could have needs on World Health Organization will access that files. The interesting factor is, there's a stress between security and therefore the different properties. The lot of we have likelihood to replicate our files, the lot of we have a tendency to introduce potential points of understanding and therefore the lot of trust we have a tendency to need. It's this stress that makes this type of drawback interesting, and provides a context within which $\mathrm{CP}-\mathrm{ABE}$ is also beneficial. Attributes of secret key are mathematically integrate into the key itself, after file is encrypted; say we put it on the server.

\section{DIAGRAM}

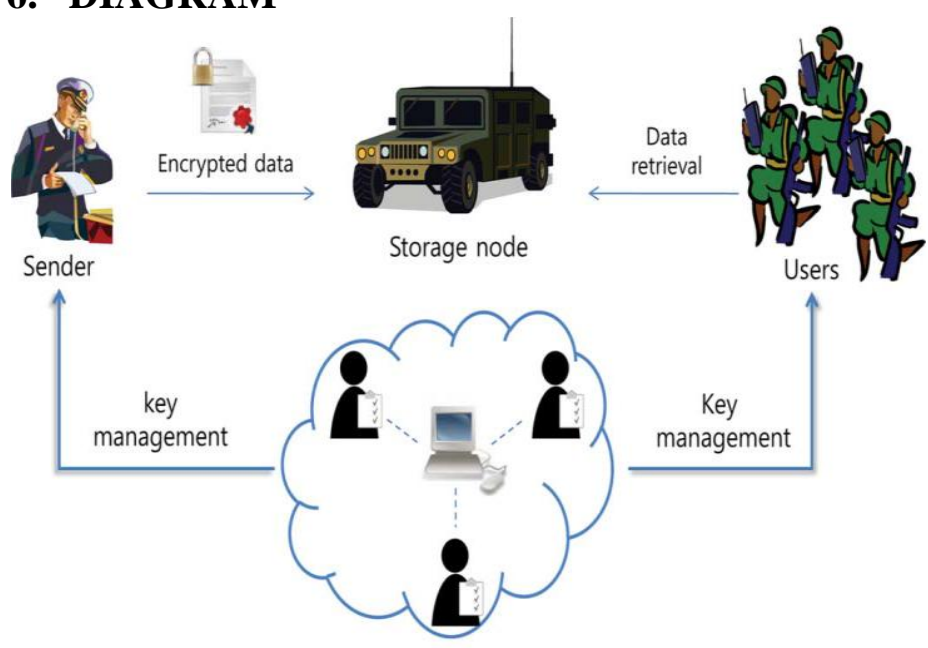

Key authorities

Fig 2: System Architecture

\section{CONCULSION}

DTN technologies are becoming successful result in military applications that permit wireless devices to communicate with each other and access the private information accurately by utilize external storage nodes. $\mathrm{CP}-\mathrm{ABE}$ is a scalable cryptographic result to the access control and reliable data retrieval issues. In this paper, we proposed an efficient and reliable data retrieval procedure using $\mathrm{CP}-\mathrm{ABE}$ for decentralized ITNs where various key authorities supervise their attributes separately. In addition, the fine-grained key revocation can be done for each attribute group. We determine how to apply the suggest mechanism to securely and efficiently manage the confidential data distributed in the interruption- tolerant military network.

\section{REFERENCES}

[1] J. Burgess, B. Gallagher, D. Jensen, and B. N. Levine, "Maxprop: Routing for vehicle-based disruption tolerant networks," in Proc.IEEE INFOCOM, 2006, pp. 1-11.

[2] M. Chuah and P. Yang, "Node density-based adaptive routing scheme for disruption tolerant networks," in Proc. IEEE MILCOM, 2006, pp. 1-6.

[3] M. M. B. Tariq, M. Ammar, and E. Zequra, "Mesage ferry route design for sparse ad hoc networks with mobile nodes," in Proc. ACMMobiHoc, 2006, pp. 37-48.

[4] S. Roy andM. Chuah, "Secure data retrieval based on ciphertext policy attribute-based encryption (CP-ABE) 
system for the DTNs," Lehigh CSE Tech. Rep., 2009.

[5] M. Chuah and P. Yang, "Performance evaluation of content-based information retrieval schemes for DTNs," in Proc. IEEE MILCOM, 2007, pp.17.

[6] M. Kallahalla, E. Riedel, R. Swaminathan, Q. Wang, and $\mathrm{K}$. Fu, "Plutus: Scalable secure file sharing on untrusted storage," in Proc. Conf. File Storage Technol., 2003, pp. $29-42$.

[7] L. Ibraimi, M. Petkovic, S. Nikova, P. Hartel, and W. Jonker,"Mediated cipher text-policy attribute-based encryption and its application," in Proc. WISA, 2009, LNCS 5932, pp. 309-323.

[8] N. Chen, M. Gerla, D. Huang, and X. Hong, "Secure, selective group broadcast in vehicular networks using dynamic attribute based encryption," in Proc. Ad Hoc
Netw. Workshop, 2010, pp. 1-8.

[9] D. Huang and M. Verma, "ASPE: Attribute-based secure policy enforcementin vehicular ad hoc networks," Ad Hoc Netw., vol. 7, no. 8, pp. 1526-1535, 2009.

[10] A. Lewko and B. Waters, "Decentralizing attribute-based encryption," Cryptology ePrint Archive: Rep. 2010/351, 2010.[11] A. Sahai and B. Waters, "Fuzzy identity-based encryption," in Proc.Eurocrypt, 2005, pp. 457-473.

[11] V. Goyal, O. Pandey, A. Sahai, and B. Waters, "Attribute-based encryption for fine-grained access control of encrypted data," in Proc.ACM Conf. Comput. Commun. Security, 2006, pp. 89-98.

[12] J. Bethencourt, A. Sahai, and B. Waters, "Ciphertextpolicy attributebased encryption," in Proc. IEEE Symp. Security Privacy, 2007, pp. 321-334. 